\section{Risk of injury after emergency department visit for acute peripheral vertigo: a matched-cohort study}

\author{
Hayoung Kim', Sihyoung Lee ${ }^{2}$, Joonghee Kim ${ }^{1}$ \\ 'Department of Emergency Medicine, Seoul National University Bundang Hospital, Seongnam, Korea \\ ${ }^{2}$ Department of Emergency Medicine, Sejong Hospital, Bucheon, Korea
}

Objective Peripheral vertigo is one of the most common causes of the emergency department (ED) visits. It can impair balance and might predispose patients to injuries after discharge. The purpose of this study was to determine whether peripheral vertigo is associated with an increased risk of trauma.

Methods This matched-cohort study used the nationally representative dataset of de-identified claim information of 1 million randomly sampled individuals from a real Korean population, from 2002 to 2013. The exposure cohort included patients who visited EDs for new-onset peripheral vertigo without prior or concurrent injury. Each patient was randomly matched to five unexposed individuals (also without previous injury) by incidence density sampling. The primary outcome was a new injury within 1 year. The secondary outcomes were various injury subtypes. The time-dependent effect of the exposure was modeled using the extended Cox model. Age, sex, comorbidities, and household income level were included as covariates.

Results A total of 776 and 3,880 individuals were included as the exposure and comparison cohorts, respectively. The risks of trunk injury and upper extremity injury were significantly higher in the exposure cohort. Extended Cox models with multivariable adjustment showed significantly increased risk for up to 1 year, with the first 1-month; 1 month to 3 months; and 3 months to 1 year hazard ratios of 5.23 (95\% confidence interval [Cl], 2.83-9.64); $1.50(95 \% \mathrm{Cl}, 1.02-2.20)$; and $1.37(95 \% \mathrm{Cl}, 1.11-1.68)$, respectively.

Conclusion Patients visiting EDs for acute peripheral vertigo are at a higher risk of a new injury for up to a year.

Keywords Dizziness; Wounds and injuries; Vertigo; Benign paroxysmal positional vertigo

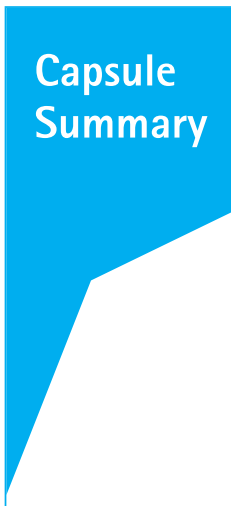

What is already known

Dizziness and vestibular asymmetry have been associated with an increased risk of falls and fractures. There is a lack of studies demonstrating an increased risk of various injury types after emergency department discharge for acute peripheral vertigo, especially with long-term perspectives.

\section{What is new in the current study}

We conducted a population-based observational study using a nationally representative cohort. We found that visiting the emergency department for acute peripheral vertigo is associated with an increased risk of injury for up to 1 year. It would be prudent to alert the patients and their family members about the risk and recommend relevant preventive measures.
elSSN: 2383-4625

Received: 15 June 2019

Revised: 22 September 2019

Accepted: 1 October 2019

Correspondence to: Joonghee Kim Department of Emergency Medicine, Seoul National University Bundang Hospital, 82 Gumi-ro 173beon-gil, Bundang-gu, Seongnam 13620, Korea E-mail: joonghee@snubh.org ORCID

https://orcid.org/0000-0001-5080-7097

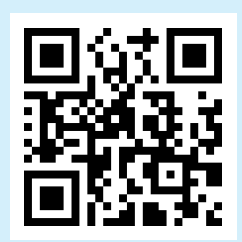

How to cite this article:

Kim H, Lee S, Kim J. Risk of injury after emergency department visit for acute peripheral vertigo: a matched-cohort study. Clin Exp Emerg Med 2020;7(3):176-182. https://doi.org/10.15441/ceem.19.064

This is an Open Access article distributed under the terms of the Creative Commons Attribution Non-Commercial License (https:// creativecommons.org/licenses/by-nc/4.0/). 


\section{INTRODUCTION}

Vertigo is a common presenting symptom in the emergency department (ED). Vertigo has both peripheral and central causes, with most vertigo cases being peripheral (inner ear) in etiologies..$^{1-3}$ Common causes of peripheral vertigo include benign paroxysmal positional vertigo (BPPV), vestibular neuritis, labyrinthitis, and Meniere disease. Peripheral vertigo is often considered benign, and patients with this diagnosis are often discharged from the ED after symptomatic treatments.

Dizziness or vertigo is one of the most common categories of symptoms for visiting the ED in the United States. ${ }^{4}$ Dizziness or vertigo is very common in the general population, with one in five people of working age and one in four elderly people age having experienced the symptom during the past month., ${ }^{5,6}$ There are more than 2 million US ED visits annually for dizziness or vertigo, comprising approximately $4.4 \%$ of all chief complaints in awake patients. ${ }^{4,7-9}$ The number of these visits increased by $90 \%$ between 1995 and 2009, and the national cost of their evaluations in the US ED was 3.9 billion US dollars per year in 2011 compared with 1.1 billion US dollars per year in 1993..$^{10,11}$

Vestibular asymmetry has been associated with an increased risk of falls and fractures. In addition, various prescriptions to control the related symptoms, such as anticholinergics, antiemetics, benzodiazepines and others, might also increase the risk of falls and fractures. ${ }^{12-16}$ Therefore, it is possible that ED visits for peripheral vertigo are associated with an increased risk of various types of injury after discharge, and this hypothetical risk may last a long time, as peripheral vertigo tends to frequently recur. However, studies demonstrating this association, especially with long-term perspectives, are lacking. Therefore, we conducted a population-based observational study using a nationally representative cohort. The purpose of the study was to determine whether a visit to an ED for a new episode of peripheral vertigo significantly increased the risk of injury.

\section{METHODS}

\section{Study design}

We conducted a matched-cohort study using a nationally representative dataset. The main exposure was an ED visit for new peripheral-type vertigo. Incidence density sampling was used to match each patient with an exposure to five unexposed individuals (also without previous injury). ${ }^{17}$ The primary outcome was a new injury event within 1 year after an ED visit. The institutional review boards of the Seoul National University Bundang Hospital approved the study (X-1705/399-904) and provided a waiver of informed consent.

\section{Data source}

The data source was the National Health Insurance Service-National Sample Cohort (NHIS-NSC) dataset released in 2015 by the Korean National Health Insurance Service (NHIS). ${ }^{18}$ The dataset was obtained from a population-based cohort with de-identified claim information including 1 million randomly sampled individuals (using stratification), from a real Korean population. The dataset contains diagnostic codes based on the Korean Classification of Diseases 6 (a Korean modification of the International Classification of Diseases-10 coding system), prescription and procedure codes, and related costs, as well as demographic information such as age, sex, and socioeconomic status from 2002 to 2013. The database also has information about disability and death based on the national disability registration data and death certificates.

\section{Definition of exposure and outcome events}

The exposure cohort included adult patients (aged 20 years or older) visiting the ED with a principal diagnosis of vertiginous disease of peripheral origin, including Meniere disease (H81.0x), BPPV (H81.1x), vestibular neuronitis (H81.2x), labyrinthitis (H83.0x), and other peripheral vertigo (H81.3x); but no new diagnosis of central vertigo ( $\mathrm{H} 81.4 \mathrm{x})$ within the week following the ED visit. The primary outcome was any new incident of injury regardless of the ED visit. The secondary outcomes were specific types of injuries, including injuries to the head and neck (S0.x, S1.x); brain (intracranial [S06.x]); trunk (thorax [S2.x] and abdomen [S3.x]); upper extremity (shoulder and upper arm [S4.X], elbow and forearm [S5.x], and wrist, hand, and fingers [S6.x]); and lower extremity (hip and thigh [S7.x], knee and lower leg [S8.x], and ankle and foot [S9.x]).

\section{Construction of cohorts and included covariates}

The study population included in this analysis was based on the following criteria. We first excluded all individuals with a diagnosis of peripheral vertigo or injury from January 1,2002, to December 31, 2003, before study entry (January 1, 2004). Among the remaining eligible participants, we built our exposure and comparison cohorts using incidence density sampling without replacement. ${ }^{17}$ Specifically, we identified the first visit of participants who visited the ED for a vertiginous disorder of peripheral origin without any previous or concurrent injury event; then, the number of days from their study entry (the day of initial diagnosis) to the first event of injury was determined. We then randomly matched each of the exposed patients to five unexposed individuals who also had no previous injury, and were alive in the NHIS cohort for at least the same period based on the age ( \pm 1 year), sex, and household income level ( \pm 1 level). Resampling was not allowed, and in- 
dividuals initially selected as an unexposed group could visit the ED for peripheral vertigo at a later time. In both cohorts, observation was censored at the time of their first injury event, death, or the end of the NHIS cohort follow-up (December 31, 2013).

The covariates considered were age, sex, household income level, and comorbidities including diabetes mellitus, hypertension, ischemic heart disease, stroke, heart failure, chronic renal failure, advanced liver disease, chronic obstructive pulmonary disorder, peripheral arterial disease, and malignancy. The presence of each of the comorbidities was defined based on the claim information of the 2-year period preceding the exposure. ${ }^{19-23}$

\section{Statistical analyses}

Categorical variables were reported using frequencies and proportions. Continuous variables were reported using mean and standard deviation or median and interquartile range, as appropriate. Wilcoxon rank-sum test, the chi-square test, or Fisher exact test was performed as appropriate for comparisons between groups.

We used an extended Cox model using time-varying coefficients to model the subsequent risk of injury. Specifically, we estimated the hazard ratios (HRs) and their 95\% confidence inter-

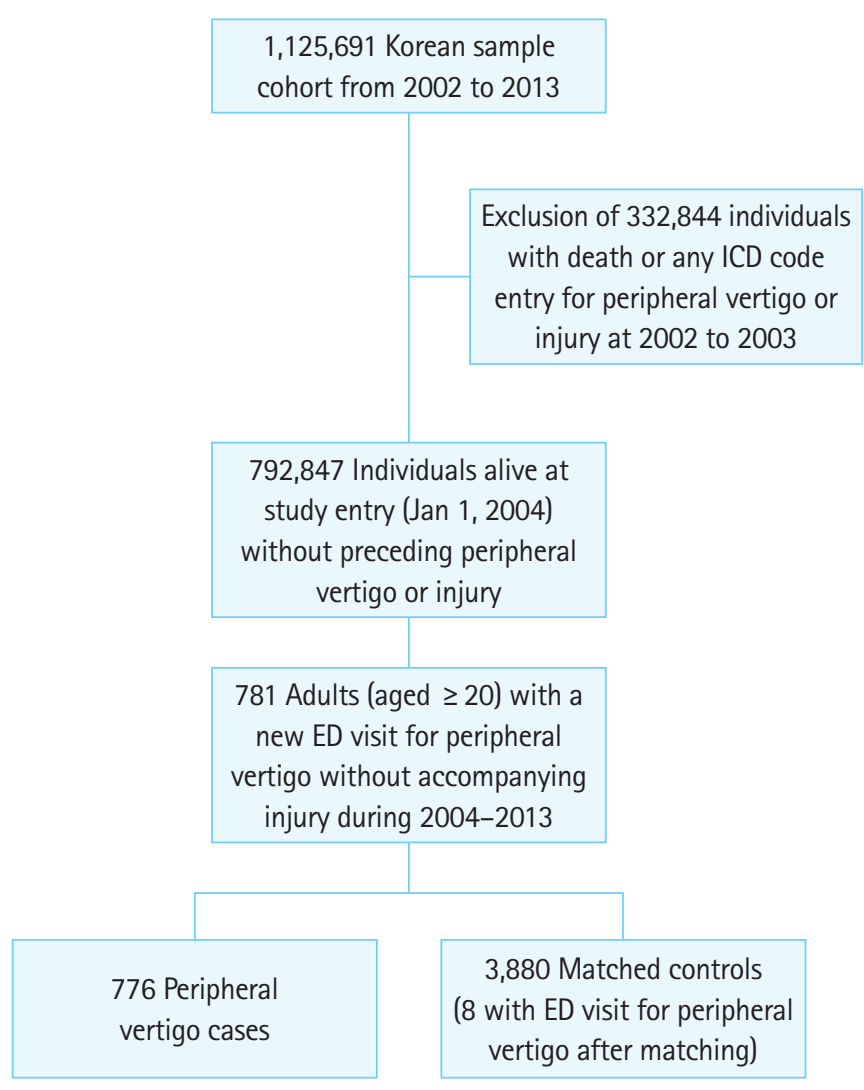

Fig. 1. The flowchart of the exposure and comparison cohorts. ICD, International Classification of Diseases; ED, emergency department. vals (Cls) for injury at three post-visit intervals (first month, 1 to 3 months, and 3 months to 1 year) to capture the diminishing effect size of acute peripheral vertigo episode. ${ }^{24}$ Using the method, we assessed the unadjusted and adjusted effect of the exposure. For the adjustment, we used two sets of covariates, one including age and sex and the other including all the covariates including age, sex, household income and whole set of comorbidities.

The goodness-of-fit of the multivariable models was tested with Gronnesby and Borgan goodness-of-fit test. The proportion-

Table 1. Baseline characteristics of study population

\begin{tabular}{|c|c|c|c|}
\hline & $\begin{array}{l}\text { Exposed } \\
(n=776)\end{array}$ & $\begin{array}{c}\text { Control } \\
(n=3,880)\end{array}$ & P-value \\
\hline \multicolumn{4}{|l|}{ Type of vertiginous disorder } \\
\hline Meniere disease (H81.0x) & $24(3.1)$ & - & \\
\hline Benign paroxysmal vertigo (H81.1x) & $417(53.7)$ & - & \\
\hline Vestibular neuronitis (H81.2x) & $135(17.4)$ & - & \\
\hline Other peripheral vertigo $(\mathrm{H} 81.3 \mathrm{x})$ & $199(25.6)$ & - & \\
\hline Labyrinthitis (H83.0x) & $1(0.1)$ & - & \\
\hline Age group (yr) & & & 0.941 \\
\hline$\leq 45$ & $257(33.1)$ & $1,290(33.2)$ & \\
\hline $46-65$ & $361(46.5)$ & $1,821(46.9)$ & \\
\hline$>65$ & $158(20.4)$ & 769 (19.8) & \\
\hline Sex, male & $269(34.7)$ & $1,345(34.7)$ & 1.000 \\
\hline Household income level & & & 0.996 \\
\hline Low & $173(22.3)$ & $863(22.2)$ & \\
\hline Mid & $251(32.3)$ & $1,250(32.2)$ & \\
\hline High & $352(45.4)$ & $1,767(45.5)$ & \\
\hline Diabetes mellitus & $46(5.9)$ & $223(5.7)$ & 0.911 \\
\hline Hypertension & $217(28.0)$ & $764(19.7)$ & $<0.001$ \\
\hline Dyslipidemia & $115(14.8)$ & $406(10.5)$ & 0.001 \\
\hline Ischemic heart disease & $52(6.7)$ & $147(3.8)$ & $<0.001$ \\
\hline Atrial fibrillation or flutter & $3(0.4)$ & $15(0.4)$ & 1.000 \\
\hline Heart failure & $15(1.9)$ & $52(1.3)$ & 0.271 \\
\hline Stroke & $28(3.6)$ & $75(1.9)$ & 0.006 \\
\hline Peripheral arterial disease & $37(4.8)$ & $132(3.4)$ & 0.080 \\
\hline Chronic renal failure & $8(1.0)$ & $18(0.5)$ & 0.095 \\
\hline Advanced liver disease & $5(0.6)$ & $11(0.3)$ & 0.218 \\
\hline Chronic obstructive pulmonary disease & $14(1.8)$ & $44(1.1)$ & 0.174 \\
\hline Malignancy & $19(2.4)$ & $87(2.2)$ & 0.826 \\
\hline
\end{tabular}

Values are presented as number (\%).

Table 2. Study outcome incidences in a year

\begin{tabular}{lccr}
\hline & $\begin{array}{c}\text { Exposed } \\
(n=776)\end{array}$ & $\begin{array}{c}\text { Control } \\
(n=3880)\end{array}$ & P-value \\
\hline Any injury & $170(21.9)$ & $577(14.9)$ & $<0.001$ \\
Head and neck injury & $37(4.8)$ & $133(3.4)$ & 0.087 \\
Trunk injury & $53(6.8)$ & $173(4.5)$ & 0.007 \\
Upper extremity injury & $57(7.3)$ & $199(5.1)$ & 0.017 \\
Lower extremity injury & $46(5.9)$ & $168(4.3)$ & 0.065
\end{tabular}

Values are presented as number (\%). 

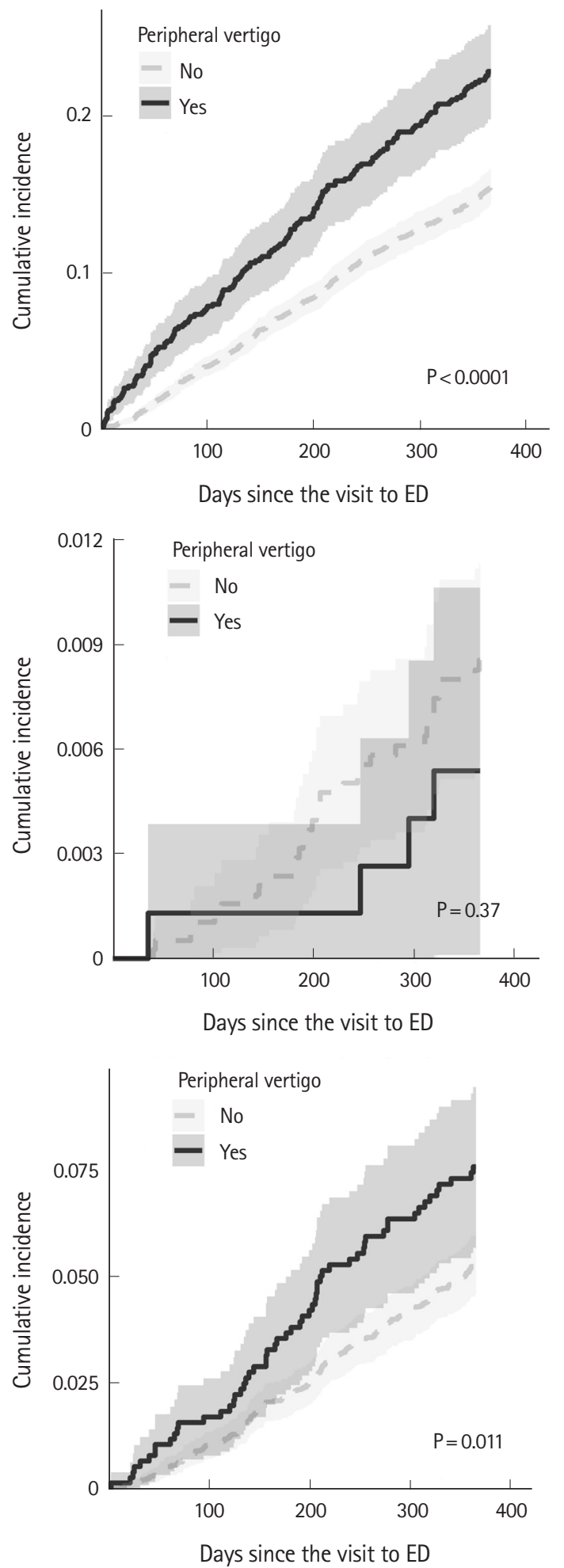
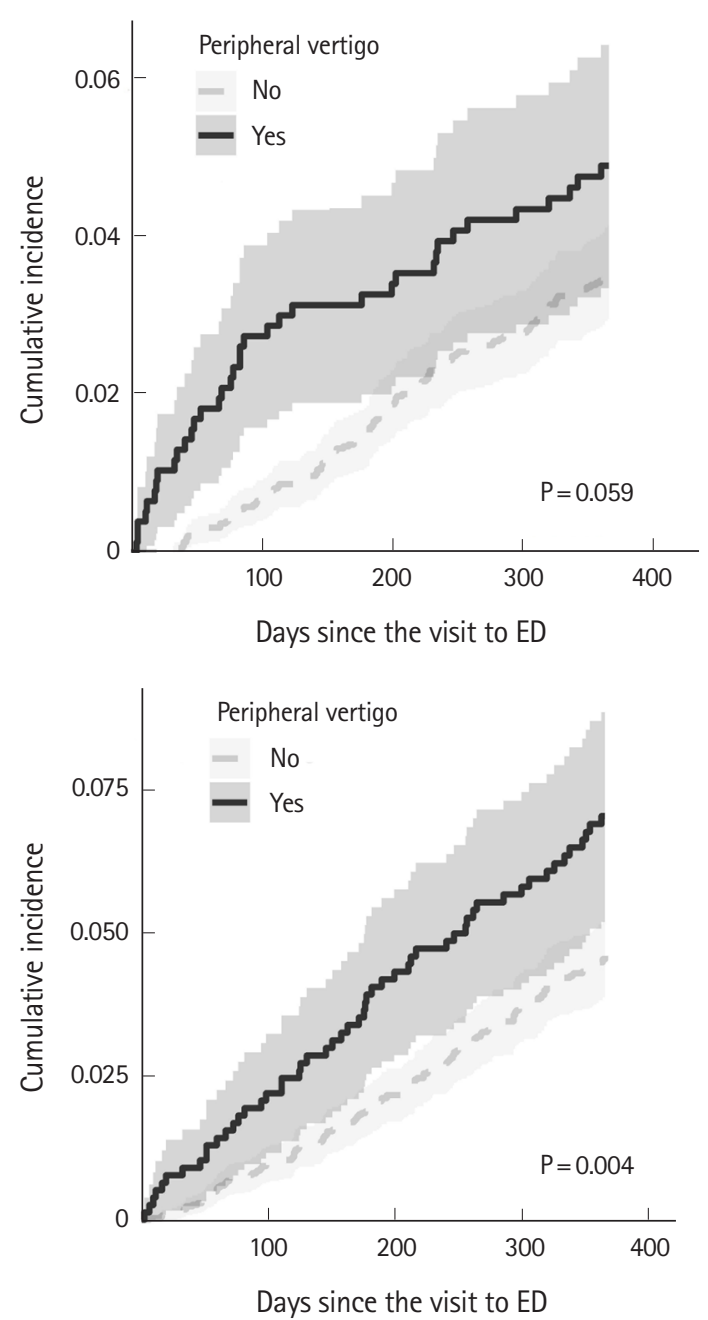

C

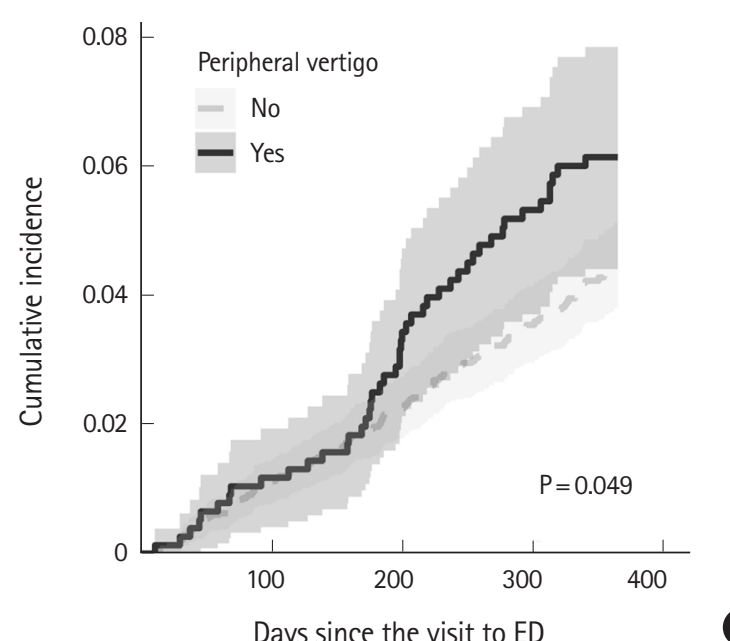

D

E

Fig. 2. The cumulative incidence of various injury events after discharge. (A) Any injury, (B) head and neck injury, (C) brain injury, (D) trunk injury, (E) upper extremity injury, and (F) lower extremity injury. ED, emergency department.

al hazard assumption was assessed by examination of Schoenfeld residual plots. The results of the Cox regression analyses were presented as HRs, with their 95\% Cls. P-values < 0.05 were con- sidered significant. All data handling and statistical analyses were performed using R-packages ver. 3.3.2 (R Foundation for Statistical Computing, Vienna, Austria). 


\section{RESULTS}

From 2004 to 2013, a total of 776 patients were included as the exposure cohort, and 3,880 patients were included as the comparison cohort (Fig. 1 and Table 1). The prevalence of hypertension, dyslipidemia, ischemic heart disease, and stroke was significantly higher in the exposure cohort $(P<0.001, P=0.001, P<0.001$, and $P=0.006$, respectively) than in the comparison cohort. The median duration of follow-up was 365 (interquartile range, 365$365)$ days for both cohorts $(P=0.623)$, with almost the same proportion of full observation over 3 years ( $n=727,93.7 \%$ vs. $n=3,653$, $94.1 \%$, respectively; $P=0.677$ ).

A total of 170 patients (21.9\%) in the exposure cohort had any injury at least once during the 1-year follow-up period (Table 2), while 577 (14.9\%) had such an event in the reference cohort $(\mathrm{P}<$ 0.001). With several types of injuries, a statistically significant difference occurred between the groups: trunk injury (53 [6.8\%] vs. $173[4.5 \%]$, respectively; $P=0.007)$; and upper extremity injury (57 [7.3\%] vs. 199 [5.1\%], respectively; $P<0.017)$. Fig. 2 shows the cumulative incidence of the first injury events during the fol-

\section{low-up period.}

The associations between peripheral vertigo and injury during the post-visit intervals (first month, 1 to 3 months, and 3 months to 1 year) were assessed using extended Cox regression. We built three regression models: 1) a univariable model; 2 ) a multivariable model including peripheral vertigo, age group, and sex; and 3) a full multivariable model including peripheral vertigo, age group, sex, household income level, and comorbidities. In all the three models, acute peripheral vertigo was associated with an increased risk of injury. The HRs in the first month were 5.33 (95\% $\mathrm{Cl}, 2.89-9.83 ; \mathrm{P}<0.001) ; 5.33$ (95\% Cl, 2.89-9.83; $\mathrm{P}<0.001)$; and $5.23(95 \% \mathrm{Cl}, 2.83-9.64 ; \mathrm{P}<0.001)$, respectively. The HRs for the 1 month to 3 months were $1.53(95 \% \mathrm{Cl}, 1.04-2.24 ; \mathrm{P}=0.030)$; $1.53(95 \% \mathrm{Cl}, 1.04-2.24 ; \mathrm{P}=0.030)$; and $1.50(95 \% \mathrm{Cl}, 1.02-2.20$; $\mathrm{P}=0.039$ ), respectively. For the 3 months to 1 year $\mathrm{HRs}$, these were $1.40(95 \% \mathrm{Cl}, 1.14-1.72 ; \mathrm{P}=0.001) ; 1.40(95 \% \mathrm{Cl}, 1.14-1.72$; $\mathrm{P}=0.001)$; and $1.37(95 \% \mathrm{Cl}, 1.11-1.68 ; \mathrm{P}=0.003)$, respectively (Table 3). Fig. 3 shows both the crude and adjusted cumulative incidence of injury in the exposure and reference cohorts during the observation. The only other variable associated with the injury

Table 3. Estimated risk of injury after exposure

\begin{tabular}{|c|c|c|c|c|c|c|}
\hline & \multicolumn{2}{|c|}{ Within 1 month } & \multicolumn{2}{|c|}{1 to 3 months } & \multicolumn{2}{|c|}{3 months to 1 year } \\
\hline & $\mathrm{HR}(95 \% \mathrm{Cl})$ & P-value & $\operatorname{HR}(95 \% \mathrm{Cl})$ & P-value & $\mathrm{HR}(95 \% \mathrm{Cl})$ & P-value \\
\hline Unadjusted & 5.33 (2.89-9.83) & $<0.001$ & $1.53(1.04-2.24)$ & 0.030 & $1.40(1.14-1.72)$ & 0.001 \\
\hline Age and sex adjusted & 5.33 (2.89-9.83) & $<0.001$ & $1.53(1.04-2.24)$ & 0.030 & $1.40(1.14-1.72)$ & 0.001 \\
\hline Full adjustment & $5.23(2.83-9.64)$ & $<0.001$ & $1.50(1.02-2.20)$ & 0.039 & 1.37 (1.11-1.68) & 0.003 \\
\hline
\end{tabular}

$\mathrm{HR}$, hazard ratio; $\mathrm{Cl}$, confidence interval.
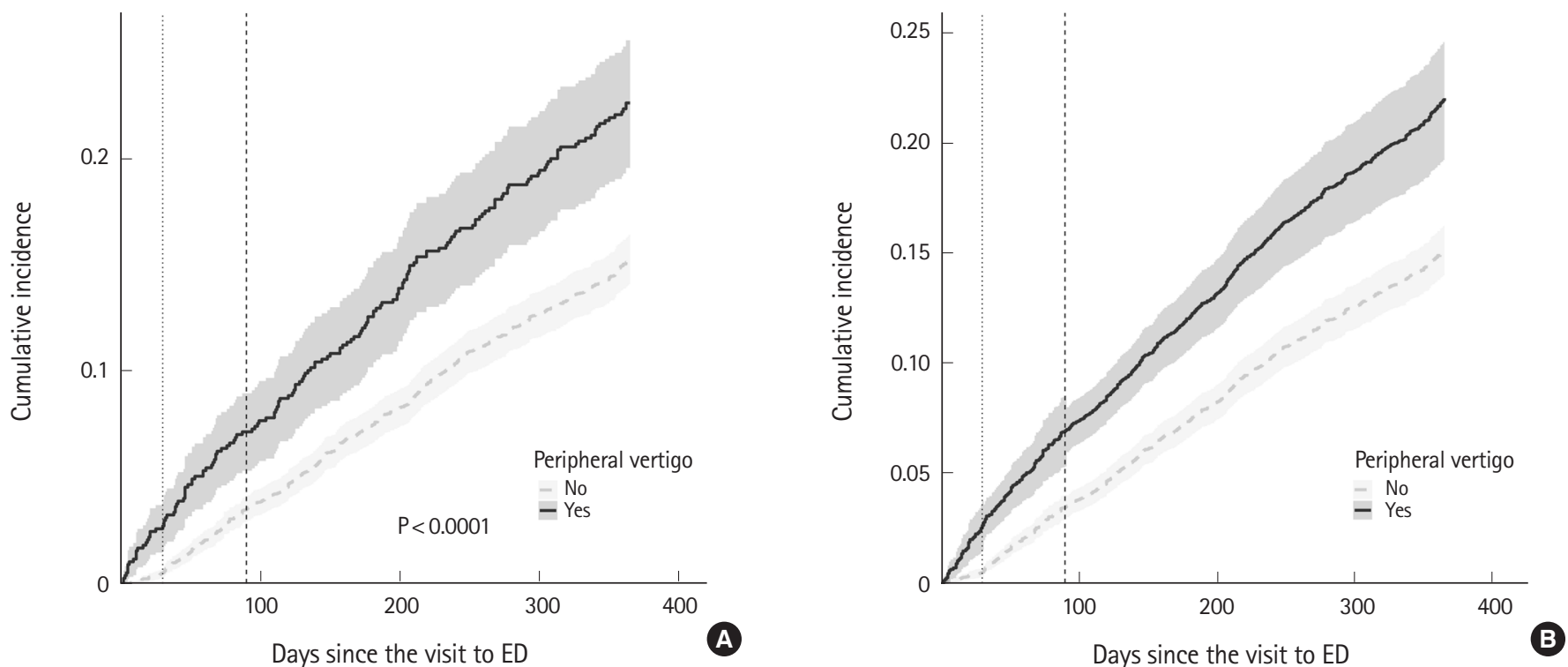

Fig. 3. Crude and adjusted cumulative incidence of injury in the exposure and reference cohorts (dotted vertical line, 30 days; dashed vertical line, 90 days). (A) Unadjusted cumulative incidence of injury and (B) adjusted cumulative incidence of injury. ED, emergency department. 
risk was history of stroke (Supplementary Tables 1, 2) with a HR of 1.53 (95\% Cl, 1.02-2.29; $P=0.039)$.

\section{DISCUSSION}

In this population-based longitudinal study, we found a new ED visit for acute peripheral vertigo to be a significant risk factor for injury for up to a year. We also found that the effect size of the exposure is time-dependent with a decreasing effect. To the best of our knowledge, this is the first observational study reporting the association between ED visits for peripheral vertigo and future risk of injury.

Current evidence on the association between peripheral vertigo and the future risk of injury is lacking. Two administrative data-based studies are currently available. The first one assessed the risk of any kind of fracture in a Taiwanese population of patients with new BPPV compared to age-, sex-, and index year-matched, randomly selected controls for up to 12 years. ${ }^{25}$ In the study, the authors observed a 1.14-fold higher risk of fracture after the diagnosis of peripheral vertigo. The second study compared the risk of accidental injuries after ED visit for peripheral vertigo to that of renal colic in a population dataset of Canadian ED visitors. $^{26}$ In contrast to the first study, no significant association was observed.

Our study demonstrated a significant increase in the risk of injury for up to a year after an ED visit for peripheral vertigo. This suggests the need for ED physicians to alert patients and their families about the risk, especially if the patient has a higher baseline risk of injury. The other risk factors considered for fall, especially in the elderly population, are frailty, vision, balance or gait problems, postural hypotension, and various medications. ${ }^{27-29}$ Recommendations of some preventive measures to prevent falls, such as improvement of the patient's environment (installation of handles and removal of tripping hazards such as slippery and uneven surfaces), physical training, medication adjustment, or proper use of assistive devices may be helpful if provided by ED physicians for patients with vertigo as well as multiple risk factors for fall. $^{29-33}$

Possibly, appropriate help from guardians might be crucial in preventing injury. However, we could not assess its effect as our dataset does not provide such details. Currently, there has been no study assessing whether such external help could significantly reduce the risk of injury. The authors hope to see a future study shedding light on this subject.

This study has several limitations. First, misclassification is a potential cause of bias in a population-based study using claims records. Secondly, the diagnosis of peripheral vertigo made in the
EDs is often not final and can often change later after the followup. Thirdly, there was no information available on symptom intensity or duration. Fourth, we did not assess the effect of medication on the outcomes because we thought the sample size of the study population was not large enough to analyze the effect of individual pharmacological variation.

Despite these limitations, the study also has some strengths. This is the first population-based analysis demonstrating that a new ED visit for peripheral vertigo is a risk factor for future injury. We analyzed data over a sufficiently long period using a statistical method that can model the time-dependency of effect size. Finally, we assessed various types of injury risks, including brain injury, which were not analyzed in previous studies.

In conclusion, visiting the ED for acute peripheral vertigo was associated with an increased risk of injury for up to a year. It would be prudent to alert the patients and their family members about the risk, and recommend relevant preventive measures.

\section{CONFLICT OF INTEREST}

No potential conflict of interest relevant to this article was reported.

\section{ACKNOWLEDGMENTS}

This study was supported by Seoul National University Bundang Hospital (SNUBH grant 13-2017-015).

\section{SUPPLEMENTARY MATERIAL}

Supplementary Tables are available from: https://doi.org/10.15441/ ceem.19.064.

\section{REFERENCES}

1. Kim AS, Fullerton HJ, Johnston SC. Risk of vascular events in emergency department patients discharged home with diagnosis of dizziness or vertigo. Ann Emerg Med 2011;57:34-41.

2. Yardley L, Owen N, Nazareth I, Luxon L. Prevalence and presentation of dizziness in a general practice community sample of working age people. Br J Gen Pract 1998;48:1131-5.

3. Tinetti ME, Williams CS, Gill TM. Dizziness among older adults: a possible geriatric syndrome. Ann Intern Med 2000;132:33744.

4. Kerber KA, Meurer WJ, West BT, Fendrick AM. Dizziness presentations in U.S. emergency departments, 1995-2004. Acad Emerg Med 2008;15:744-50. 
5. Newman-Toker DE, Hsieh YH, Camargo CA Jr, Pelletier AJ, Butchy GT, Edlow JA. Spectrum of dizziness visits to US emergency departments: cross-sectional analysis from a nationally representative sample. Mayo Clin Proc 2008;83:765-75.

6. Newman-Toker DE, Cannon LM, Stofferahn ME, Rothman RE, Hsieh YH, Zee DS. Imprecision in patient reports of dizziness symptom quality: a cross-sectional study conducted in an acute care setting. Mayo Clin Proc 2007;82:1329-40.

7. Newman-Toker DE, Camargo CA Jr, Hsieh YH, Pelletier AJ, EdIow JA. Disconnect between charted vestibular diagnoses and emergency department management decisions: a cross-sectional analysis from a nationally representative sample. Acad Emerg Med 2009;16:970-7.

8. Saber Tehrani AS, Coughlan D, Hsieh YH, et al. Rising annual costs of dizziness presentations to U.S. emergency departments. Acad Emerg Med 2013;20:689-96.

9. Baloh RW, Honrubia V, Jacobson K. Benign positional vertigo: clinical and oculographic features in 240 cases. Neurology 1987;37:371-8.

10. Cappello $M$, di Blasi $U$, di Piazza $L$, et al. Dizziness and vertigo in a department of emergency medicine. Eur J Emerg Med 1995;2:201-11.

11. Karatas M. Central vertigo and dizziness: epidemiology, differential diagnosis, and common causes. Neurologist 2008;14: 355-64.

12. Shih RD, Walsh $B$, Eskin $B$, et al. Diazepam and meclizine are equally effective in the treatment of vertigo: an emergency department randomized double-blind placebo-controlled trial. J Emerg Med 2017;52:23-7.

13. Management of benign paroxysmal positional vertigo. Drug Ther Bull 2009;47:62-6.

14. Strupp M, Kremmyda O, Brandt T. Pharmacotherapy of vestibular disorders and nystagmus. Semin Neurol 2013;33:286-96.

15. Sacco RR, Burmeister DB, Rupp VA, Greenberg MR. Management of benign paroxysmal positional vertigo: a randomized controlled trial. J Emerg Med 2014;46:575-81.

16. Cesarani A, Alpini D, Monti B, Raponi G. The treatment of acute vertigo. Neurol Sci 2004;25 Suppl 1:S26-30.

17. Wang $M H$, Shugart $Y Y$, Cole SR, Platz EA. A simulation study of control sampling methods for nested case-control studies of genetic and molecular biomarkers and prostate cancer progression. Cancer Epidemiol Biomarkers Prev 2009;18:706-11.

18. Lee J, Lee JS, Park SH, Shin SA, Kim K. Cohort profile: the National Health Insurance Service-National Sample Cohort (NHISNSC), South Korea. Int J Epidemiol 2017;46:e15.

19. Kang SH, Kim J, Park JJ, et al. Risk of stroke in congestive heart failure with and without atrial fibrillation. Int J Cardiol 2017;
248:182-7.

20. Kim B, Kim J, Jo YH, et al. Risk of pneumonia after vertebral compression fracture in women with low bone density: a population-based study. Spine (Phila Pa 1976) 2018;43:E830-5.

21. Kim H, Kim J, Kim S, et al. Cardiovascular effects of long-term exposure to air pollution: a population-based study with 900 845 person-years of follow-up. J Am Heart Assoc 2017;6.

22. Lee $\mathrm{S}$, You CY, Kim J, et al. Long-term cardiovascular risk of hypertensive events in emergency department: a populationbased 10-year follow-up study. PLoS One 2018;13:e0191738.

23. Kim $B$, Jeong $H$, Kim J, et al. Incidence and risk factors of delayed intracranial hemorrhage in the emergency department. Am J Emerg Med 2018;36:271-6.

24. Therneau T, Crowson C, Atkinson E. Multi-state models and competing risks [Internet]. [place unknown]: CRAN; 2020 [cited 2019 Sep 4]. Available from: https://cran.r-project.org/ web/packages/survival/vignettes/compete.pdf.

25. Liao WL, Chang TP, Chen HJ, Kao CH. Benign paroxysmal positional vertigo is associated with an increased risk of fracture: a population-based cohort study. J Orthop Sports Phys Ther 2015;45:406-12.

26. Atzema CL, Grewal K, Lu H, Kapral MK, Kulkarni G, Austin PC. Outcomes among patients discharged from the emergency department with a diagnosis of peripheral vertigo. Ann Neurol 2016;79:32-41.

27. Cummings SR, Melton L. Epidemiology and outcomes of osteoporotic fractures. Lancet 2002;359:1761-7.

28. Dunlop DD, Manheim LM, Sohn MW, Liu X, Chang RW. Incidence of functional limitation in older adults: the impact of gender, race, and chronic conditions. Arch Phys Med Rehabil 2002;83:964-71.

29. Ambrose AF, Paul G, Hausdorff JM. Risk factors for falls among older adults: a review of the literature. Maturitas 2013;75:5161.

30. Gillespie LD, Gillespie WJ, Robertson MC, Lamb SE, Cumming $\mathrm{RG}$, Rowe BH. Interventions for preventing falls in elderly people. Cochrane Database Syst Rev 2003;(4):CD000340.

31. Li F, Harmer $P$, Fisher $K J$, et al. Tai Chi and fall reductions in older adults: a randomized controlled trial. J Gerontol A Biol Sci Med Sci 2005;60:187-94.

32. Campbell AJ, Robertson MC, Gardner MM, Norton RN, Buchner DM. Psychotropic medication withdrawal and a homebased exercise program to prevent falls: a randomized, controlled trial. J Am Geriatr Soc 1999;47:850-3.

33. Soriano TA, DeCherrie LV, Thomas DC. Falls in the communitydwelling older adult: a review for primary-care providers. Clin Interv Aging 2007;2:545-54. 